\title{
Research of Logistics Cost Based on Saving Algorithm: A Case of A Certain Logistics Company's Logistics Cost
}

\author{
Jing NIE',a,Wei Yuan ZENG ${ }^{1}$ Hong Lei JING ${ }^{1}$ \\ ${ }^{1}$ Department of Software Engineering, Xiamen Institute of Software Technology, Xiamen,361024, China.
}

\begin{abstract}
In recent years, logistics companies of different sizes has been considerably developed all over the country, with the intensified competitions. To stand out in the competitive market, a logistics company must improve its logistics situation, focus on the function of distribution center, reduce logistics costs for enhancing the impact of supply chain, and rapidly response to customer needs. Thus, how to better design or optimize the existing distribution route for reducing logistics costs becomes a difficult problem, and this article will do some researches on it.

Keywords: logistics company; distribution route; logistics costs; saving algorithm
\end{abstract}

\section{Introduction}

With the huge business opportunity and potential of modern logistics industry, it attracts China's attention more significantly. In 2009, the State Council issued the Adjustment and Plan for Logistics Industry, setting a milestone in the history of China's economic development. With the publication of this government document, the "logistics craze" occurred in domestic market: all kinds of new logistics companies were launched and the existing logistics companies accelerated the adjustments of their own long-term plan. Now in the international market, the share of logistics costs in GDP is supposed to symbolize the strength of a country. Although from 1991 to 2001 the share of China's logistics costs in GDP has dropped from $24 \%$ to $19.4 \%$, by 4.6 percentage points, it is still unacceptable that from 2002 to 2009 that has fallen from $18.8 \%$ to $18.1 \%$, by only 0.7 percentage points. However, the logistics costs of developed countries remain $10 \%$ in general, obviously indicating that China's logistics costs still have room for further reduction. According to saving algorithm, this article will combine with a certain case and put forward a countermeasure with actual value and operability to save logistics costs.

\footnotetext{
a Corresponding author: 56564995@QQ.COM
}

\section{Problem Description and Modeling}

\subsection{Problem Description}

Let goods be delivered from Distribution Center $\mathrm{O}$ to Transfer Station (Customer) $v_{i}(i=1, \cdots, m): \quad q_{i}$ $(i=1, \cdots, m)$ is the volume of goods delivery from $\mathrm{O}$ to $v_{i}(i=1, \cdots, m)$, and $L_{i j}(\mathrm{i}, \mathrm{j}=1, \ldots, \mathrm{m})$ is the delivery distance between $p_{i}$ and $p_{j}$; as delivery vehicles are classified into $\mathrm{n}$ categories by load (or capacity), there are $x_{i}(\mathrm{i}=1, \ldots, \mathrm{n})$ delivery vehicles with $w_{i}$, where $w_{i-1}<$ $w_{i}$ and $w_{i}>\sum_{i=1}^{m} q_{i}$,i.e. the load (or capacity) of each delivery vehicle outweighs the total customer demands.

Thus, if without any limitations, goods should be delivered by a vehicle, transforming the issue into a shortest route problem.

In this article, the conditions of route optimization are defined to be that when a logistics distribution center delivers goods to multiple customers (or transfer stations), the locations of logistics center and customers are given; the distance among logistics center and customers (or transfer stations)and among different customers (or transfer stations) are given and fixed. Meanwhile, the traffic limit on transportation network is out of 
consideration; customer time demand is out of limitation; each customer demand for goods does not exceed the maximum load of delivery vehicles while the goods can be mixed in a same vehicle; each vehicle hasa fixed maximum load, so overload cannot be allowed; each vehicle is set out from logistics centerand back here after delivery task is completed.

\subsection{Parameter Definition and Problem Model}

V: Customer (or transfer station) set

$\mathrm{O}$ : Logistics distribution center

W: Capacity of delivery vehicle

$q_{i}:$ Customer (or transfer station) demand

$c_{i j}$ : Distance between customer (or transfer station) and another customer (or transfer station)

$y_{i k}: 1$ represents that Vehicle $\mathrm{k}$ is driven from customer $\mathrm{i}$ to Customer j; 0 represents that Vehicle $\mathrm{k}$ is not driven from customer $\mathrm{i}$ to Customer $\mathrm{j}$

$x_{i j k}: 1$ represents that Customer $\mathrm{i}$ is served by Vehicle $\mathrm{k} ; 0$ represents that Customer $\mathrm{i}$ is not served by Vehicle $\mathrm{k}$.

So mathematical modeling can be as follows:

$$
\begin{array}{r}
\min \sum_{k=1}^{K} \sum_{i=1}^{V} \sum_{i \neq j, j=0}^{V} c_{i j} x_{i j k}(1) \\
\text { st. } \sum_{i=0}^{V} q_{i} y_{i k} \leq W, \forall k \subseteq K(2) \\
\sum_{k=1}^{K} y_{i k}=\left\{\begin{array}{ll}
k, & i=0 \\
l, & i \subseteq V
\end{array}(3)\right. \\
\sum_{i \in V} x_{i j k}=y_{j k}, \forall j \in V, k \in K(4) \\
\sum_{j \in V} x_{i j k}=y_{j k}, \forall i \in V, k \in K(5) \\
x_{i j k} \in\{0,1\}, \forall i, j \in V, k \in K(6) \\
y_{j k} \in\{0,1\}, \forall i \in V, k \in K(7)
\end{array}
$$

The meanings of equation(1) to equation(7) are explained as follows:

Equation(1): Find the minimum of route distance sum Equation(2): Total volume of goods through each route does not exceed the capacity of delivery vehicles

Equation(3): Upper equation represents the number of vehicles in logistics distribution center; lower one represents that each demand site can only be served by a vehicle

Equation(4): Traffic conservation limit

Equation(5): Traffic conservation limit

Equation(6): Distribution site of Vehicle k

Equation(7): Route of Vehicle k

\section{Algorithm Analysis}

This article chooses C-W saving algorithm to optimize the distribution of a certain logistics company, due to the fact that in logistics distribution system route optimization has too many limits including vehicles' capacity limits, load limits, time limits, etc., while saving algorithm has the advantage of adding constraints flexibly.

\subsection{Distribution Model}

\subsubsection{Point-to-point Transportation}

Shortest route algorithm between two points is the most basic one in the category of logistics distribution. It determines the best route among multiple distribution sites by calculating the shortest route between two points. Given the actual constraints such as the one-way traffic system and delivery speed, as well as the road control on vehicle types, etc., the road network within distribution area can be taken as a Digraph $\mathrm{G}=(\mathrm{V}, \mathrm{E})$ with weights, where the weight of each side is a non-negative real number. In addition, let the point of $\mathrm{v}$ be a starting point of distribution. Then calculate the shortest route from the starting point to other vertexes.

\subsubsection{Single-loop Transportation - TSP}

Single-loop transportation problem is to select an appropriate and closure-required route through all the nodes for a given node-set $\mathrm{D}$ in the route optimization. In transportation decision, it mainly aims at the route arrangement of a single vehicle for ensuring that the shortest distance is driven by the target vehicle through all the customers. TSP mathematical model is described as:

Based on the Digraph $\mathrm{G}=(\mathrm{V}, \mathrm{E})$, where $V=\left\{v_{1}, v_{2}, \cdots, v_{n}\right\}, E=\left\{e_{1}, e_{2}, \cdots, e_{m}\right\}$

$$
\begin{gathered}
C=\left\{c_{i j} \mid i, j \in N, 1 \leq i, j \leq n\right\} \\
\text { Distance is } D=\left\{d_{i j} \mid i, j \in N, 1 \leq i, j \leq n\right\} \\
\text { Objective function is } \min \sum_{i=1}^{n} c_{i(i+1)} d_{i(i+1)}
\end{gathered}
$$

The traveling salesman sets out from and returns to the starting point, thus $v_{n+1}=v_{1}$.

\subsubsection{Multi-loop Transportation - VRP}

Multi-loop transportation is a common distribution issue in societyand an integration of single-loop transportation problems. It originates from TSP. VRP is generally defined as an appropriated route arrangement that makes vehicles pass a series of loading point and (or) unloading point orderly and achieve some certain purposes (such as the shortest distance, least cost, shortest time, least vehicles, etc.) under the constraints (such as the demands for goods, delivery volumes, delivery times, vehicles' capacity limits, distance limits and time limits, etc.).

VRP mathematical model is described as:

Based on the Digraph $G=(V, E)$, the vertex set is $V=\left\{v_{0}, v_{1}, \cdots, v_{n}\right\}$, the set of two vertexes(or side set) is $E=\left\{\left(v_{i}, v_{j}\right) \mid v_{i}, v_{j} \in V, i \neq j\right\}$, where $v_{0}$ represents the 
location of distribution center, and other nodes represent all the customers.

The basic condition of a typical VRP model is arranging $\mathrm{N}$ vehicles to deliver goods for L customers. Its purpose is to determine the number of vehicle $M$ $(\mathrm{M} \leq \mathrm{N})$ and to finish the arrangement of loop transportation with the minimum costs. Its target model can be represented as follows:

Objective function: $\min Z=\sum_{m=1}^{M} \sum_{l=1}^{L} d_{l m} x_{l m}$

Constraints:

$$
\begin{gathered}
\sum_{l=1}^{L} w_{l} x_{l m} \leq b_{m},(l=1,2, \cdots, L ; m=1,2, \cdots, M) \\
\sum_{m=1}^{M} x_{l m}=L,(m=1,2, \cdots, M) x_{l m} \in\{0,1\},
\end{gathered}
$$

$(l=1,2, \cdots, L ; m=1,2, \cdots, M)$

where $b_{m}$ is the transport capacity of Vehicle m; $\mathrm{L}$ is the sum of customers; $\mathrm{M}$ is the sum of vehicles; $w_{l}$ is the demand (by weight or volume) of Customer 1.

\subsection{Shortest Route Solution of Point-to-point Transportation - Dijkstra Algorithm}

Dijkstra algorithm is also called dual labeling method. This method is to give the connecting Point $v_{j}$ two labels $l_{j}, k_{j}$ in the Digraph $\mathrm{G}=(\mathrm{V}, \mathrm{E})$, where the first label $l_{j}$ represents the shortest distance from the starting point $v_{s}$ to $v_{j}$ and the second one $k_{j}$ represents the subscript of one adjacent point before $v_{j}$ in this shortest route, further finding the shortest route.

\subsection{Basic Principle of Saving Algorithm}

Saving algorithm is put forward by Clarke and Wright in 1964, aiming at solving vehicle routing problem. Its basic principle is: Distribution Center $\mathrm{O}$ delivers goods to $\mathrm{n}$ distribution sites through one-node route connecting all the sites and distribution center. Therefore, the total cost of distribution transportation is:

$$
z=\sum_{i=1}^{n} c_{o i}+\sum_{i=1}^{n} c_{i o}
$$

If Distribution Site $i$ is connected with Site $j$ and forms a route tothe distribution center, the saving cost will be:

$$
\begin{aligned}
& s(i, j)=c_{o i}+c_{i o}+c_{o j}+c_{j o}-\left(c_{o i}+c_{i j}+c_{j o}\right) \\
& =c_{i o}+c_{o j}-c_{i j}
\end{aligned}
$$

The more the value of $s(i, j)$ is, the more costs will save when connecting Site $\mathrm{i}$ and Site $\mathrm{j}$. In the practical route optimization, saving cost is sorted by the value of $\mathrm{s}(\mathrm{i}, \mathrm{j})$ in descending order. This algorithm has an advantage of fast calculation speed. Especially in the small-scale distribution optimization, the calculation is completed fast by the close accuracy and best solution.

\section{Calculation Example}

As the distribution center, a certain logistics company delivers goods to 12 customers. The center has five vehicles with the load of 680 items per vehicle. Find the best solution for the shortest route.

This issue for VRP with single center and non-full loads has the following characteristics: the location of logistics distribution center is given and single; the locations and demands of transfer stations are given; the route and distance among transfer stations are given and determined; vehicles are only unloaded in transfer stations and return empty to the distribution center after distribution; the distribution center has only a sort of vehicles. Find the objective function: the minimum cost of vehicle delivery routes is reflected as the shortest distance in this solution.

According to the data provided by a certain logistics company, draw a route distribution from this company to each transfer station at last, as shown below:

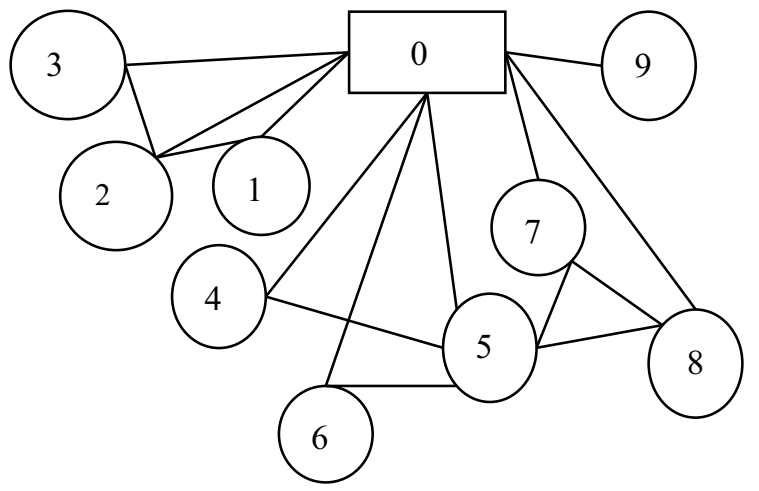

Figure 1. Route distribution from this company to each transfer station

\subsection{Symbol Instructions of Transfer Stations}

Table 1. Symbol Correspondences of Transfer Stations

\begin{tabular}{|c|c|c|c|c|c|c|c|c|}
\hline$p_{1}$ & $p_{2}$ & $p_{3}$ & $p_{4}$ & $p_{5}$ & $p_{6}$ & $p_{7}$ & $p_{8}$ & $p_{9}$ \\
\hline 1 & 2 & 3 & 4 & 5 & 6 & 7 & 8 & 9 \\
\hline
\end{tabular}

Table 2. Demands of Transfer Stations (Unit: Item)

\begin{tabular}{|l|c|c|c|c|c|c|c|c|}
\hline$p_{1}$ & $p_{2}$ & $p_{3}$ & $p_{4}$ & $p_{5}$ & $p_{6}$ & $p_{7}$ & $p_{8}$ & $p_{9}$ \\
\hline 470 & 690 & 625 & 300 & 525 & 230 & 295 & 225 & 295 \\
\hline
\end{tabular}

\subsection{Solution Process.}


First, calculate the distances among this company and transfer stations.

Second, calculate the shortest distances among this company and transfer stations.

Third, calculate the saving mileage $s(i, j)$ among customers by saving algorithm, according to the shortest distances.

Forth, sort $s(i, j)$ in descending order.

Fifth, find the initial solution.
Sixth, find the best solution (according to the savings in descending order).

According to the saving algorithm, we can find that only the route below can save the distance. The saving distance is as shown below:

Table 3. Route and Distance

\begin{tabular}{|c|c|c|c|c|c|c|c|}
\hline Route & $p_{5}, p_{6}$ & $p_{4}, p_{6}$ & $p_{6}, p_{8}$ & $p_{4}, p_{8}$ & $p_{6}, p_{7}$ & $p_{4}, p_{7}$ & $p_{7}, p_{8}$ \\
\hline Distance & $5-6$ & $4-6$ & $6-8$ & $4-8$ & $6-7$ & $4-7$ & $7-8$ \\
\hline Mileage & 2433 & 2438 & 2443 & $>2438$ & $>2438$ & $>2438$ & $>2438$ \\
\hline
\end{tabular}

By the comparative analysis of the table above, we find that Route 5-6 can save the maximum mileage under the premise of equal vehicles.

According to the practical route, the saving mileages of Route 5-6 in practice is less than that in theory. Therefore, we should choose the route of the second maximum saving mileage, i.e. Route 4-6.

Theoretically, the goods delivery from logistics center to nine transfer stations respectively needs 10 vehicles once and 20 vehicles twice. According to the current situation of this company, goods are delivered to transfer stations twice per week basically. The working time of vehicles is from Tuesday to Saturday, i.e. five days per week. If goods are delivered to nine transfer stations twice in the five days, 18 transfer stations can be reached; and if five vehicles are on work in the five days, 25 places (transfer stations) can be reached. Therefore, the existing five vehicles with full load can achieve the annual freight volume by the route above, completing the task with the excess capacity of solving emergencies.

Compared with original route, this route saves costs as follows:

goods are delivered 2 per week and 8 times per month at most.

Monthly saving mileages $(\mathrm{km}): 218 \times 8=1744$

Monthly saving tolls (yuan): $25 \times 8=160$

Monthly saving oil costs (yuan): $1744 \times 0.33 \times 6.7=3856$

(Logistics vehicle of this company costs $0.33 \mathrm{~L}$ oil per kilometer, and oil price is calculated by 6.7 yuan/L.)

Annual saving oil costs (yuan): $3856 \times 12=46272$

Annual saving tolls (yuan): $160 \times 12=1920$

Total saving costs (equaling annual saving oil costs plus annual saving tolls): $46272+1920=48192$.

\section{Acknowledgments}

Fund Project: the Project of Xiamen Science and Technology Program under Grant Nos. 3502Z20153006, 3502Z20133042.

\section{References}

1. Lv Xiongwei.Researches on post VRPTW based on genetic algorithm [J].Journal of Shandong University: Engineering Science, 2009, 44(6):46-50.

2. LIU Cheng.VRP with interval parameters and its improved C-W algorithm [J].Journal of Wuhan University of Technology, 2010, 32(2):182-185.

3. FAN Zhan, Liang Guolong.Design and expending of adaptive neighborhood searching algorithm for TSP [J].Computer Engineering and Applications, 2008, 44(12):71-74.

4. Zhang Xuezhi, Chen Gongyu.An improved saving method of the vehicle routing problem [J].Systems Engineering, 2008, 26(11):67-70.

5. Altinel 1,Oncan T.A new enhancement of the Clarke and Wright savings heuristic for the capacitated vehicle routing problem[J].The Journal of the 
Operational Research Society,2005,56(8):954-961.

6. 2008International Conference on Wireless Communications.A Modified Max-min Ant System for Vehicle Routing Problems[C].Dalian[Zhao Gang, Luo Wenjuan,et al],2008:1-4.

7. Barbarosoglu G,Ozgur D.A tabu search algorithm for the vehicle routing
problem[J].Computers\&Operations

Research,1999,26(3):255-270.

8. Remy Spliet, Guy Desaulniers. The discrete time window assignment vehicle routing problem [J].European Journal of Operational Research.2015.8:1-13. 